\title{
Elaboración de Indicadores Específicos de Idoneidad Didáctica en Probabilidad: Aplicación para la Reflexión sobre la Práctica
} Docente

\author{
Elaboration of Specific Didactical Suitability Criteria in Probability: \\ Application for Reflection on the Teaching Practice
}

\author{
Pablo Beltrán-Pellicer* \\ ORCID iD 0000-0002-1275-9976 \\ Juan D. Godino** \\ ORCID iD 0000-0001-8409-0258 \\ Belén Giacomone*** \\ ORCID iD 0000-0001-6752-2362
}

\begin{abstract}
Resumen
En este artículo se presenta la elaboración de una guía de valoración de la idoneidad didáctica (GVID) para el estudio de la probabilidad en educación secundaria. El objetivo que se persigue es disponer de un instrumento que promueva la reflexión docente en torno a experiencias de enseñanza-aprendizaje de un contenido concreto. El método de investigación toma como punto de partida la revisión sistemática de los conocimientos didácticomatemáticos de cada una de las facetas en las que se descompone un proceso educativo: epistémica-ecológica, cognitiva-afectiva e instruccional (interaccional y uso de medios tecnológicos). Posteriormente, se aplica la GVID elaborada a una experiencia didáctica con alumnos de educación secundaria. Los resultados de dicha aplicación revelan el potencial de esta herramienta para facilitar la reflexión sobre la propia práctica, establecer relaciones entre las distintas facetas e identificar posibles mejoras en el diseño en ciclos sucesivos.
\end{abstract}

Palabras clave: Idoneidad Didáctica. Conocimientos Didáctico-Matemáticos. Enseñanza de la Probabilidad. Práctica Reflexiva.

\begin{abstract}
This paper introduces the development of a didactic suitability assessment guide (DSAG) for probability studies in secondary education. The objective is to have an instrument that promotes teacher reflection about teaching and learning experiences within a specific content. The starting point which the research method takes is the

\footnotetext{
* Doctor por la Universidad Nacional de Educación a Distancia (UNED). Profesor asociado en la Universidad de Zaragoza, España. Dirección postal: Área de Didáctica de la Matemática, Facultad de Educación, c/ Pedro Cerbuna, 12, Zaragoza, España, CP: 50009. E-mail: pbeltran@unizar.es.

** Doctor por la Universidad de Granada (UGR). Profesor catedrático en la Universidad de Granada (UGR), Granada, España. Dirección postal: Departamento de Didáctica de la Matemática, Facultad de Ciencias de la Educación, Campus de Cartuja, s/n, Granada, España, CP: 18071. E-mail: jgodino@ugr.es.

**** Maestría por la Universidad de Granada (UGR). Doctoranda en la Universidad de Granada (UGR), Granada, España. Dirección postal: Campus de Cartuja, s/n, Departamento de Didáctica de la Matemática, Facultad de Ciencias de la Educación. Granada, España, CP: 18071. E-mail: giacomone @ correo.ugr.es.
} 
systematic review of the didactic-mathematical knowledge of each of the facets in which an educational process is decomposed: epistemic-ecological, cognitive-affective, and instructional (interactional and use of technological means). Subsequently, the DSAG is applied to an educational experience with students of secondary education. The results of this application reveal this tool's potential to streamline the reflection on the practice itself, to establish relationships between the different facets, and to identify possible improvements in the design in successive cycles.

Keywords: Didactical Suitability. Didactic-Mathematical Knowledge. Secondary Education. Reflective Practice.

\section{Introducción}

Aunque no se pueden esperar de las didácticas especiales recetas generales para la enseñanza de los contenidos curriculares que indiquen qué y cómo enseñar en cada circunstancia, es razonable pensar que de los esfuerzos de investigación se deriven resultados que orienten y ayuden a los profesores en las tareas docentes. Este es uno de los supuestos subyacentes de la Teoría de la Idoneidad Didáctica (TID) (GODINO, 2013; GODINO et al., 2006), mediante la cual se propone sistematizar principios, indicadores o criterios, sobre los que existe un consenso en la comunidad educativa de un campo específico (matemáticas, ciencias etc.), cuya aplicación podría ayudar a alcanzar niveles altos de idoneidad de los procesos instruccionales.

En la TID se proponen seis facetas o dimensiones para el análisis de los procesos instruccionales, identificando, para cada faceta, criterios de idoneidad generales (GODINO, 2013), de aplicación a cualquier contenido matemático. De esta forma, se dispone de una guía general de indicadores de idoneidad (GVID) que puede ser un instrumento de ayuda para el profesor, tanto en las fases de diseño como de implementación y evaluación de procesos instruccionales. Ahora bien, sobre los procesos de enseñanza y aprendizaje de cada contenido específico (estructuras aditivas, números racionales, proporcionalidad, derivada etc.) existen múltiples investigaciones y resultados que aportan criterios específicos para el logro de procesos instruccionales de alta idoneidad (ALSINA; DOMINGO, 2010; ARGUEDAS; CONCARI; GIACOMONE，2017; AROZA; GODINO; BELTRÁN-PELLICER，2016; BLANCO-ÁLVAREZ; FERNÁNDEZ-OLIVERAS; OLIVERAS， 2017; CRUZ; GEA; GIACOMONE, 2017; CRUZ et al., 2017; ROBLES; TELLECHEA; FONT, 2014).

Resulta pertinente, por lo tanto, la elaboración de guías específicas para los distintos contenidos curriculares, que particularicen y desarrollen la GVID. Este es un objetivo de interés para la investigación en didáctica, ya que tales guías específicas pueden ser usadas por los profesores como instrumentos de apoyo para la reflexión sobre la práctica docente. 
En este artículo se presenta el proceso de construcción de una GVID en torno a la probabilidad y su aplicación por un profesor como instrumento de reflexión sobre una experiencia de enseñanza de la probabilidad en educación secundaria. Se pretende, también, mostrar la potencial utilidad de la TID en la formación de profesores.

\section{Problema, marco teórico y método}

Se trata de introducir una metodología para realizar la reconstrucción de un significado de referencia para el estudio de la probabilidad, en las diversas facetas: epistémica y ecológica, cognitiva y afectiva e instruccional (interaccional y uso de medios tecnológicos). Para afrontar esto, se realiza un análisis del contenido de investigaciones clave sobre los distintos aspectos de la enseñanza y aprendizaje de la probabilidad en educación secundaria, orientado por la herramienta teórica de la idoneidad didáctica. Este análisis tendrá como objetivo extraer indicadores específicos de idoneidad en cada faceta y cada componente, cuya expresión genérica sería de la forma:

El proceso de estudio, en las circunstancias contextuales fijadas, debería tener en cuenta (...) para que se pueda afirmar que tiene una idoneidad didáctica alta.

No se trata de reglas generales de actuación, sino de ciertos principios cuya aplicación requiere tener en cuenta las restricciones del contexto y los recursos disponibles.

\subsection{La noción de idoneidad didáctica: facetas, componentes e indicadores}

La idoneidad didáctica de un proceso de instrucción se define como el grado en que dicho proceso (o una parte del mismo) reúne ciertas características que permiten calificarlo como óptimo o adecuado para conseguir la adaptación entre los significados personales logrados por los estudiantes (aprendizaje) y los significados institucionales pretendidos o implementados (enseñanza), teniendo en cuenta las circunstancias y recursos disponibles (entorno). Esto supone la articulación coherente y sistémica de seis facetas o dimensiones (GODINO; BATANERO; FONT, 2007): idoneidad epistémica, ecológica, cognitiva, afectiva, interaccional y mediacional.

Diversas tendencias sobre la formación de profesores, tanto inicial como continua, proponen la reflexión sobre la práctica docente como una competencia clave para el desarrollo profesional y la mejora de la enseñanza (GELLERT; BECERRA; CHAPMAN, 2013; 
PARADA; PLUVINAGE, 2014; POCHULU; FONT; RODRÍGUEZ, 2016).

Entre dichas tendencias destacan la investigación-acción, la práctica reflexiva y el estudio de lecciones, desarrollado en Japón y difundido en diversos países. La investigaciónacción es un método de investigación cualitativa que se basa, fundamentalmente, en convertir en centro de atención lo que ocurre en la actividad docente cotidiana, con el fin de descubrir qué aspectos pueden ser mejorados o cambiados para conseguir una actuación más satisfactoria (ELLIOT, 1990). De acuerdo con Bradbury-Huan (2010, p. 98), "Los investigadores en la acción planifican ciclos de acción y reflexión y por tanto deben ser reflexivos sobre cómo los esfuerzos de cambio se despliegan y el impacto que nuestra intervención está teniendo".

Heredera de la investigación-acción es la corriente promovida por Schön (1983, p. 281), quien desarrolla su teoría de la práctica reflexiva en la cual aboga por un docente que reflexione, de modo permanente, sobre su práctica de enseñanza con el fin de transformarla. Schön describió la reflexión como "una continua interacción entre el pensamiento y la acción"; y presenta al práctico reflexivo como la persona que "reflexiona sobre las comprensiones implícitas en la propia acción, que las hace explícitas, las critica, reestructura y aplica en la acción futura" (SCHÖN, 1983, p. 50).

Por su parte, el estudio de clases (lesson studies) se refiere a la metodología desarrollada en Japón para la enseñanza de las matemáticas (basada en la resolución de problemas), la formación y perfeccionamiento de los profesores y está teniendo un fuerte impacto a nivel internacional como se muestra en Hart, Alston y Murata (2011). Los profesores involucrados en la enseñanza de la misma clase, o unidad didáctica, se observan unos a otros, sucesivamente, mientras la imparten, poniendo en común sus observaciones entre una clase y la siguiente, como base para tomar decisiones colectivas sobre cambios posteriores en las programaciones de las clases, que se pondrán a prueba posteriormente en la siguiente sesión.

En estas tendencias se trata de promover la reflexión del profesor, bien de manera personal o en interacción con otros colegas. Sin embargo, dada la multiplicidad de disciplinas para las cuales se proponen y la generalidad con la cual se aborda el tema de la reflexión sobre la acción, no se encuentran instrumentos explícitos que guíen la reflexión profesional sistemática en el campo específico de la educación matemática.

Sin dudar de la utilidad, incluso necesidad, de que el profesor adopte una actitud reflexiva sobre su propia práctica, y de las indudables potencialidades de compartir tales 
reflexiones en el seno de comunidades profesionales, nos parece que tales esfuerzos se verían reforzados si los profesores compartieran algunas herramientas conceptuales para apoyar sus reflexiones de manera sistemática y detallada.

En este sentido la TID, puede ser una de tales herramientas generadas a partir de un consenso en la educación matemática, conforme se explica en Breda, Font y Pino-Fan (2018). La GVID resume los componentes e indicadores de idoneidad y se ofrece como un recurso de apoyo para la reflexión del profesor en las fases de diseño, implementación y evaluación de sus experiencias de enseñanza.

\title{
3 Indicadores de idoneidad didáctica de procesos de estudio de la probabilidad
}

\subsection{Faceta epistémica}

De acuerdo con Godino (2013, p. 118-119)

\begin{abstract}
Un proceso de estudio matemático tiene mayor idoneidad epistémica en la medida en que los significados institucionales implementados (o pretendidos) representan bien a un significado de referencia. Dicho significado de referencia será relativo al nivel educativo en el que tiene lugar el proceso de estudio y deberá ser elaborado teniendo en cuenta los diversos tipos de problemas y contextos de uso del contenido objeto de enseñanza, así como las prácticas operativas y discursivas requeridas.
\end{abstract}

Según esto, el primer paso que se debe dar es identificar o reconstruir el significado de referencia de la probabilidad en el contexto educativo correspondiente, por ejemplo, educación secundaria obligatoria (estudiantes de 12-16 años).

La probabilidad, en los niveles educativos de primaria y secundaria, se puede abordar desde tres enfoques, subjetivo, frecuencial y clásico, cada uno de los cuales comporta sistemas de prácticas operativas y discursivas diferentes y, por tanto, significados parciales diferentes (BATANERO, 2005). El estudio de estos tres significados debe ir precedido de un significado informal, ligado al uso cotidiano de la palabra probabilidad y otros términos sinónimos y asociados, como se muestra en Godino, Batanero y Cañizares (1987).

Un proceso de estudio diseñado o implementado se puede calificar con mayor idoneidad epistémica en la medida en que están representados y articulados estos significados. A continuación, resumimos los elementos característicos de estos significados, siguiendo básicamente el texto de Batanero y Godino (2002), junto con algunas consideraciones propias de la faceta ecológica. 


\subsubsection{Significado informal (uso cotidiano del lenguaje)}

El lenguaje cotidiano incorpora multitud de términos relacionados con los fenómenos aleatorios. Es natural, por lo tanto, que el alumnado haya elaborado una interpretación personal de dichos significados, a partir de la cual se debe fundamentar la secuencia didáctica. Analizamos, a continuación, cada una de las categorías de objetos matemáticos desde este significado informal:

- Situaciones-problema. Deberían contemplarse situaciones en las que se discutan el uso cotidiano de términos ligados a las situaciones estocásticas de una variedad de contextos (GODINO et al., 1987, p. 19-20). Un ejemplo sencillo consiste en lanzar un debate en el aula acerca del tiempo atmosférico que hará dentro de unas horas, anotando en el cuaderno algunas de las respuestas, para disponer de esa referencia posteriormente. Además, resulta fundamental introducir, de forma explícita, la diferencia entre fenómenos aleatorios y deterministas; con situaciones que tienen que ver con alguna ley física determinista. Por ejemplo, si dejo caer esta moneda, ¿caerá al suelo?

- Lenguajes. Las situaciones esbozadas anteriormente se sustentan en el lenguaje verbal, en el que se enmarcan términos de uso cotidiano como posible, previsible, presumible, factible, viable, probable, verosímil, incierto, seguro etc.

- Conceptos y definiciones. Una vez que se ha dado ocasión de poner en práctica los significados personales, es conveniente institucionalizar las definiciones de fenómeno aleatorio y fenómeno determinista. Por otro lado, la diversidad de términos empleados permite establecer una gradación informal en la escala de probabilidad, acudiendo para ello a los diccionarios de la lengua.

- Proposiciones y propiedades. En este caso, se trata de proposiciones informales en las que se indica la mayor o menor probabilidad de ocurrencia de sucesos mediante el empleo del término correspondiente del lenguaje común.

- Procedimientos. El principal procedimiento que se enmarca dentro del significado informal consiste en la asignación de la palabra más adecuada para expresar el grado de probabilidad de un suceso. Los matices de cada uno de los términos ocasionarán que este procedimiento no proporcione un único resultado final.

- Argumentos y justificaciones. Los argumentos justifican las proposiciones y los procedimientos. De esta manera, cada vez que se elige un término para describir un fenómeno aleatorio se puede invitar a poner en común las razones de dicha elección. Por 
otro lado, son sumamente importantes los argumentos en torno a la diferenciación de fenómenos aleatorios y deterministas, puesto que relacionan las principales definiciones de este primer estadio de significados con la institucionalización de los conceptos y las reglas utilizadas.

\subsubsection{Significado subjetivo}

El significado subjetivo de la probabilidad consiste en cuantificar los resultados posibles de un fenómeno aleatorio a partir de experiencias personales previas y de su contraste con las condiciones en que tiene lugar dicho fenómeno. Este significado se ve ampliado, más adelante, con la introducción de la inferencia bayesiana. Sin embargo, en los niveles propios de este trabajo, nos quedamos simplemente con la asignación subjetiva de probabilidades.

- Situaciones-problema. En Batanero y Godino (2002) se describe una situación idónea para introducir la asignación subjetiva de probabilidades referida al pronóstico del tiempo. A partir de un listado de términos de uso informal, se invita a su gradación de menor a mayor confianza y se propone como tarea buscar esas expresiones en los medios. Lo interesante, ahora, es introducir la noción de probabilidad como fracción, de manera que esa gradación de términos vaya acompañada de una estimación en la escala de probabilidad entre 0 y 1 , o las fracciones equivalentes correspondientes; lo cual hace la diferencia respecto al significado informal.

- Lenguajes. De nuevo entran en juego términos y expresiones del lenguaje cotidiano, como cierto, posible, bastante probable, hay alguna posibilidad, seguro, es imposible, casi imposible, se espera que, incierto, hay igual probabilidad, puede ser, sin duda etc. A esta lista, debemos añadir los términos ya comentados de experimento aleatorio, suceso, experimento determinista, grado de creencia en la ocurrencia de un suceso, suceso seguro y suceso imposible. Finalmente, se incorpora un nuevo registro semiótico (Figura 1) la escala de probabilidad, intervalo numérico real entre 0 y 1.

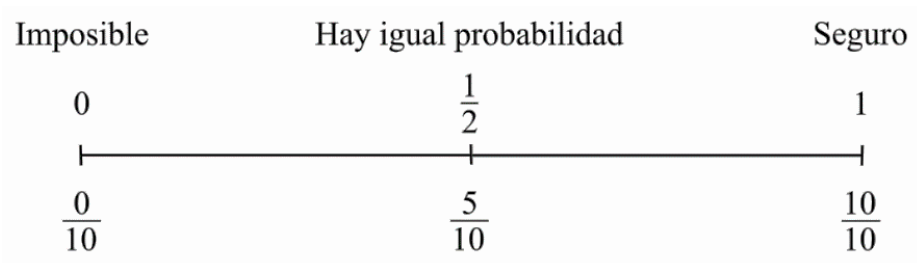

Figura 1 - Escala de probabilidad

Fuente: elaborado por los autores 
- Conceptos y definiciones. Reglas de uso de las expresiones mencionadas, en particular, grado de creencia en la ocurrencia de un suceso; suceso seguro; imposible.

- Proposiciones y propiedades. La probabilidad de un suceso es un número entre 0 y 1.

- Procedimientos. Asignación de mayor o menor posibilidad de ocurrencia (probabilidad) de un suceso con palabras y expresiones modales; asignación de probabilidades con números comprendidos entre 0 y 1 .

- Argumentos y justificaciones. Como se ha convenido en asignar el valor 0 al suceso imposible y 1 al suceso seguro, a cualquier otro suceso de un experimento se le debe asociar un valor menor que 1 y mayor que 0 .

\subsubsection{Significado frecuencial}

El significado frecuencial permite articular, de forma coherente, los contenidos de probabilidad en este nivel curricular. Precede al significado clásico, puesto que es necesario cuantificar esas experiencias aleatorias que se introducían ya de manera informal o subjetiva, y para ello será preciso elaborar tablas de frecuencias y establecer conclusiones en torno a los datos recogidos.

- Situaciones-problema. Las situaciones donde emerge de forma directa el significado frecuencial de los objetos probabilísticos consisten en experimentos reales y simulaciones. En los experimentos reales, el alumno se ve inmerso en sistemas de prácticas que, de forma guiada, permiten llevar a cabo una realimentación en términos informativos de lo que está ocurriendo. Por ejemplo, los lanzamientos de dados o monedas formarían parte de estas situaciones, con la elaboración de su correspondiente tabla de frecuencias. Las simulaciones con aplicaciones informáticas van a permitir un número mayor de repeticiones del experimento, permitiendo comprobar, empíricamente, la estabilidad de las frecuencias relativas.

Los experimentos reales y las simulaciones conforman un punto de articulación de significados de suma importancia. Las vivencias personales de cada individuo no proporcionan, normalmente, información útil para desarrollar el pensamiento probabilístico, ya que los resultados experimentales que proporcionan se diluyen en la cantidad de repeticiones necesarias. Por esta razón, como veremos, es común la aparición de sesgos en el razonamiento y creencias erróneas.

- Lenguajes. Inicialmente, los términos informales del lenguaje cotidiano siguen estando 
presentes en estas situaciones. Por otro lado, en el significado subjetivo ya se introdujo el registro numérico, como escala de probabilidad, que ahora debemos enriquecer con otras representaciones numéricas, en forma de fracción, como frecuencia relativa o porcentaje. Por otro lado, la elaboración de tablas de frecuencias permite resumir los resultados en registros gráficos, como histogramas y diagramas de barras.

- Conceptos y definiciones. La definición en torno a la cual se apoyan el resto de objetos matemáticos, desde este significado de la probabilidad, es la frecuencia relativa de un suceso. Definiciones auxiliares que deben ponerse en juego son las de frecuencia absoluta y frecuencia acumulada.

- Proposiciones y propiedades $(\mathrm{P})$ :

- P1: La frecuencia relativa del suceso varía entre 0 y 1.

- P2: La frecuencia relativa del suceso seguro siempre es 1 en cualquier serie de ensayos.

- P3: Supongamos que un suceso A se forma uniendo sucesos que no tienen elementos comunes. En este caso, la frecuencia relativa del suceso A es la suma de las frecuencias relativas de los sucesos que lo componen.

○ P4: La serie de frecuencias relativas de un suceso obtenida a medida que el número de veces que se realiza un experimento aleatorio aumenta tiende a aproximarse a un valor fijo.

- Procedimientos. Estimación frecuencial de la probabilidad, a partir del registro de los resultados de un experimento.

- Argumentos y justificaciones (A):

○ A1: La frecuencia relativa siempre está entre 0 y 1, debido a que es una fracción en la que el denominador siempre es mayor o igual que el numerador.

- A2: La frecuencia relativa del suceso seguro es 1 porque el numerador de la fracción es igual al denominador. Es decir, es el suceso que siempre ocurre.

- A3. La justificación de P3 se puede basar en experiencias que muestren el carácter sumable de la magnitud discreta número de elementos de una colección.

- A4: La observación de largas series de ensayos permite comprobar empíricamente que la frecuencia relativa tiende a un número determinado. 


\subsubsection{Significado clásico o a priori}

La concepción clásica va ligada a los juegos de azar, como expresan Cardano (1961/1663), en su Liber de Ludo Aleae, y Fermat y Pascal (1963/1654), en su célebre correspondencia en torno al problema del jugador. Se parte del supuesto de que todos los sucesos elementales son equiprobables, y se entiende la probabilidad como la fracción de casos favorables entre casos posibles. Es decir, además de la noción de equiprobabilidad, se asume que el conjunto de sucesos posibles es finito. Esto es válido para muchas aplicaciones, pero genera obstáculos epistemológicos con los que más tarde habrá que trabajar (cuando los resultados posibles sean infinitos o no se pueda calcular su probabilidad a priori), cosa que en sí no es perniciosa, porque gran parte del aprendizaje significativo se construye resolviendo esos obstáculos.

- Situaciones-problema. Las situaciones que se planteen, especialmente al principio, deben diseñarse con dos objetivos. En primer lugar, que resulte sencillo predecir la probabilidad de cada suceso y, en segundo lugar, que pueda llevarse a cabo una experiencia real o una simulación. No es trivial, desde el punto de vista epistémico, enlazar la visión a priori con la frecuencial y, por ello, se han de plantear este tipo de situaciones duales cuando se introduce el nuevo significado. Ejemplos de situaciones son los lanzamientos de una, dos o tres monedas, lanzamientos de dados, extracciones de bolas dentro una urna etc.

- Lenguajes. A los términos informales del lenguaje cotidiano, la representación numérica y los gráficos estadísticos, ya mencionados anteriormente, el significado clásico añade nuevos registros, como el esquemático (diagrama de árbol) y el simbólico (conjuntista).

- Conceptos y definiciones. Los sistemas de prácticas desde los que emerge este significado requieren de la institucionalización de una serie de conceptos a partir de sus definiciones. Se trata del espacio muestral, del suceso simple y suceso compuesto y de la equiprobabilidad de sucesos.

- Proposiciones y propiedades. La proposición fundamental que subyace en el significado clásico es la regla de Laplace; es decir, que la probabilidad de un suceso es igual a la fracción de casos favorables entre los casos posibles.

- Procedimientos. Desglosar el espacio muestral, de forma sistemática, para obtener todos los resultados posibles; emplear técnicas básicas de combinatoria para contar los casos posibles y favorables; construir el diagrama de árbol para un experimento aleatorio.

- Argumentos y justificaciones. El principio de simetría o indiferencia de los sucesos 
elementales permite asignar a cada uno de ellos la misma probabilidad. Es decir, no existe ningún argumento físico que favorezca unos sucesos frente a otros, de manera que, si hay n sucesos, la probabilidad de cada uno de ellos es $1 / \mathrm{n}$.

En el Cuadro 1 se sintetizan los indicadores de idoneidad epistémica específicos para los procesos de estudio de la probabilidad, según los componentes propuestos en Godino (2013).

\begin{tabular}{|c|c|}
\hline Componentes & Indicadores \\
\hline $\begin{array}{l}\text { Situaciones- } \\
\text { problema }\end{array}$ & $\begin{array}{l}\text { 1) Se plantean situaciones-problema que muestran y relacionan los diferentes } \\
\text { significados de la probabilidad (informal, subjetiva, frecuencial y clásica). } \\
\text { 2) Se propone una muestra representativa de experiencias aleatorias, reales o virtuales, } \\
\text { distinguiéndolas de experiencias deterministas. Por ejemplo: lanzamientos de dados } \\
\text { o monedas, simulaciones de concursos o bingos etc. } \\
\text { 3) Se propone una muestra representativa de contextos donde ejercitar y aplicar los } \\
\text { contenidos tratados. } \\
\text { 4) Se proponen situaciones de generación de problemas sobre fenómenos aleatorios } \\
\text { (problematización) por los propios estudiantes. }\end{array}$ \\
\hline Lenguajes & $\begin{array}{l}\text { 1) Se emplean diferentes registros y representaciones para describir experiencias } \\
\text { aleatorias (verbal, diagrama de árbol, tablas, simbólica, conjuntos etc.), señalando } \\
\text { las relaciones entre las mismas. } \\
\text { 2) Se utiliza un nivel lingüístico adecuado al alumnado al que se dirige, en cuanto a } \\
\text { construcciones gramaticales y vocabulario. } \\
\text { 3) Se emplean términos precisos, como suceso, espacio muestral, frecuencia relativa, } \\
\text { aleatorio, determinista, casos favorables, casos totales, resultado de un experimento, } \\
\text { sucesos simples y sucesos compuestos. } \\
\text { 4) Se proponen situaciones de expresión matemática e interpretación de fenómenos } \\
\text { aleatorios, en los diferentes registros mencionados. }\end{array}$ \\
\hline $\begin{array}{l}\text { Reglas } \\
\text { (definiciones, } \\
\text { proposiciones, } \\
\text { procedimientos) }\end{array}$ & $\begin{array}{l}\text { 1) Las definiciones y procedimientos se formulan con claridad y corrección, adaptados } \\
\text { al nivel educativo al que se dirigen. } \\
\text { 2) Se presentan las definiciones de fenómeno aleatorio, fenómeno determinista, } \\
\text { espacio muestral, suceso, suceso elemental, suceso compuesto y probabilidad. } \\
\text { 3) Se presentan proposiciones en torno a las definiciones, como la probabilidad del } \\
\text { suceso imposible, del suceso seguro y del complementario; propiedades de las } \\
\text { frecuencias relativas } \\
\text { 4) Estabilidad de las frecuencias relativas como base para estimar la probabilidad. } \\
\text { 5) Se presentan los procedimientos de cálculo de probabilidades mediante la regla de } \\
\text { Laplace y el empleo de tablas y diagramas de árbol. } \\
\text { 6) Se proponen situaciones donde los alumnos tengan que generar o negociar } \\
\text { definiciones, proposiciones o procedimientos. }\end{array}$ \\
\hline Argumentos & $\begin{array}{l}\text { 1) Las explicaciones, comprobaciones y demostraciones son adecuadas al nivel } \\
\text { educativo al que se dirigen. } \\
\text { 2) Se usan simulaciones para mostrar la estabilidad de las frecuencias relativas. } \\
\text { 3) Se promueven situaciones donde el alumno tenga que argumentar. }\end{array}$ \\
\hline Relaciones & $\begin{array}{l}\text { 1) Los objetos matemáticos (problemas, definiciones, proposiciones etc.) se relacionan } \\
\text { y conectan entre sí. } \\
\text { 2) Se identifican y articulan los diversos significados de la probabilidad (uso informal, } \\
\text { subjetivo, frecuencial y clásico). }\end{array}$ \\
\hline
\end{tabular}

Cuadro 1 - Indicadores específicos para la idoneidad epistémica en probabilidad Fuente: elaborado por los autores

\subsection{Faceta ecológica}

La secuencia didáctica no constituye un ente aislado, pues su finalidad es ser 
implementada en un centro educativo concreto, con un contexto específico. Por ello, la idoneidad ecológica considera cuatro aspectos, según los cuales se valora el grado de adecuación al entorno, tanto normativo (adaptación al currículo), como social. Dentro del entorno social entran en juego, a su vez, diferentes factores, entre los que identificamos la adaptación socio-profesional y cultural y la educación en valores. Todo ello conlleva una capacidad de adaptación que, en términos didácticos, se corresponde con la innovación didáctica.

A pesar de que podría mencionarse algún aspecto concreto, como el tratamiento preventivo de la ludopatía con actividades de aula no centradas exclusivamente en el conocimiento de la probabilidad (WILLIAMS; CONNOLLY, 2006), los indicadores ecológicos son esencialmente generales. Es decir, habrá que considerar la normativa curricular de cada institución, aspectos socioculturales y de innovación, y conexiones con otras disciplinas. Por ejemplo, los contextos para la alfabetización en probabilidad que cita Gal (2005, p. 53) deben ser tenidos en cuenta en el diseño de las actividades.

\subsection{Facetas cognitiva y afectiva}

Batanero y Sánchez (2005) señalan la especificidad del razonamiento probabilístico, frente al lógico, el numérico o el causal, debido a que es muy fácil toparse a niveles elementales con resultados que no tienen nada que ver con la intuición.

En segundo ciclo de secundaria, los alumnos aceptan con facilidad la idea de suceso equiprobable, aunque no son conscientes de las implicaciones matemáticas de tal afirmación. Si bien son capaces de comparar fracciones y aplicar el mismo razonamiento de proporcionalidad para decidir qué sucesos son más probables que otros, una correcta aproximación tendría que estar basada en la teoría axiomática de Kolmogorov.

Por ello, a pesar de que la aproximación frecuencial presenta dificultades tanto desde el punto de vista epistemológico, como desde el conceptual y práctico, resulta la más adecuada en esta etapa del desarrollo cognitivo. Estos obstáculos se deben a que realmente nunca se llega a conocer el valor exacto de la probabilidad de un suceso, porque el número de repeticiones o ensayos va a estar siempre limitado de forma práctica. Sin embargo, se pueden diseñar experiencias de aula sencillas o utilizar simulaciones por ordenador para solventar esta limitación.

Cada uno de los significados mencionados, debe tenerse en cuenta a la hora de diseñar 
herramientas para la evaluación. En este sentido, es especialmente relevante el trabajo de Green (1982) sobre la evaluación del razonamiento probabilístico en niños y adolescentes, quien diseñara un instrumento en tres partes claramente diferenciadas: combinatoria, verbal y probabilística. La inclusión de preguntas específicas para evaluar el razonamiento puramente combinatorio se justifica a partir de las teorías de Piaget e Inhelder (1951), las cuales indican que es preciso haber desarrollado y madurado previamente las estructuras operatorias del pensamiento formal.

Por otro lado, diversos trabajos (KONOLD, 1989, 1991; LECOUTRE, 1992; SERRANO et al., 1998; TVERSKY; KAHNEMAN, 1974) han tratado de caracterizar y categorizar los sesgos de razonamiento probabilístico y las dificultades más comunes en torno a estas nociones. De esta manera, en la heurística de la representatividad (TVERSKY; KAHNEMAN, 1974) se incluyen el sesgo de insensibilidad al tamaño de la muestra y las concepciones erróneas sobre secuencias aleatorias, como la falacia del jugador. Por otro lado, el sesgo de equiprobabilidad se pone de manifiesto fácilmente, según una experiencia descrita en las investigaciones sobre la cuestión (LECOUTRE; DURAND, 1988; LECOUTRE, 1992). $\mathrm{Y}$, finalmente, hay personas que presentan un enfoque en el resultado aislado (KONOLD, 1991). Además, la influencia del dominio afectivo en la resolución de problemas se ha constatado en diversas investigaciones (BLANCO; GUERRERO; CABALLERO, 2013) haciendo evidente que la afectividad y la cognición están inexorablemente interconectadas (HANNULA et al., 2005). En educación matemática, es habitual hablar de emociones, actitudes y creencias, división propuesta por McLeod (1988). El hecho de estar tratando contenidos probabilísticos no modifica la GVID original de GODINO (2013).

El Cuadro 2 recoge los indicadores de la idoneidad específicos para la faceta cognitiva, a partir de los conocimientos didáctico-matemáticos tratados en esta sección. Nótese que, respecto a la GVID original (GODINO, 2013), únicamente se modifican los indicadores relativos a los conocimientos previos. Los que tratan de valorar las adaptaciones curriculares a las diferencias individuales y el aspecto evaluativo del aprendizaje se omiten. 


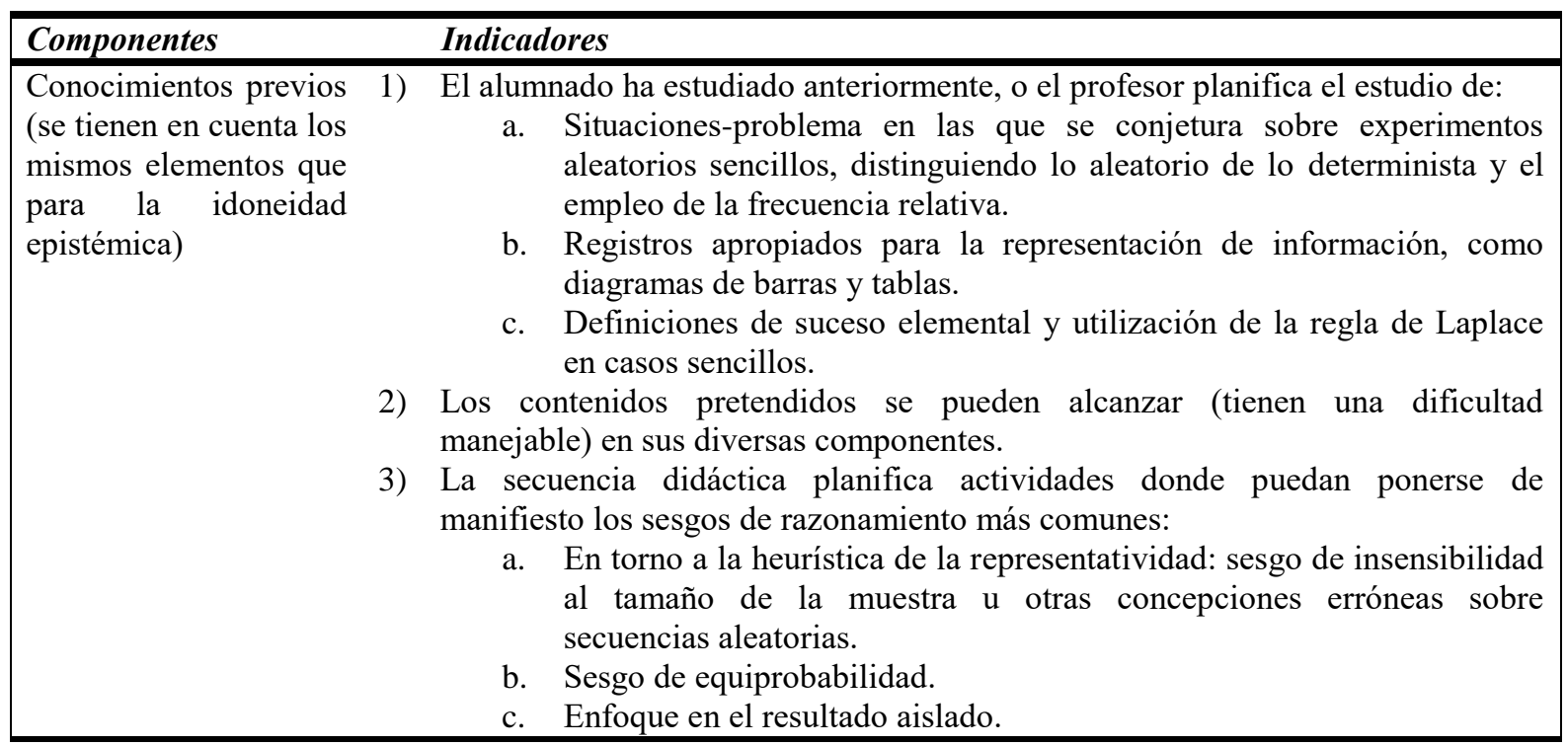

Cuadro 2 - Indicadores específicos para la idoneidad cognitiva en probabilidad Fuente: elaborado por los autores

\subsection{Faceta instruccional (interaccional y mediacional)}

Godino et al. (2006) reconocen el papel fundamental que juegan las interacciones en el proceso de instrucción, pilares del Interaccionismo Simbólico (COBB; BAUERSFELD, 1995) y de la Teoría de las Situaciones Didácticas (BROUSSEAU, 1997), escuelas de pensamiento entre las que se identifican claras correspondencias.

La importancia de los medios empleados en cualquier ámbito de la educación matemática es indudable. En un reciente trabajo de síntesis en educación estocástica, Batanero (2015) reivindica la importancia de articular los diferentes significados de la probabilidad y de plantear el aprendizaje desde las creencias e intuiciones propias de los estudiantes. Es más, en el centro de las interacciones debe situarse una experimentación o simulación, a partir de la cual pueda argumentarse y refutar o afianzar ciertas concepciones frente a otras.

En el caso de la enseñanza de la probabilidad, el uso de materiales manipulativos e informáticos, introducido como indicador general, se puede particularizar haciendo mención al uso del material probabilístico descrito en Godino et al. (1987, p. 175), y simuladores virtuales de experiencias aleatorias cada vez más abundantes y disponibles en internet.

Asimismo, el papel que deberían jugar la experimentación, el modelado y las simulaciones en los puntos de articulación de significados resulta esencial (CHAPUT; GIRARD; HENRY, 2011). Son precisamente las series largas de ensayos, facilitadas por 
aplicaciones de software, las que permiten dotar de una base empírica a la estabilidad de las frecuencias relativas.

\section{Aplicación de la GVID sobre una experiencia de enseñanza-aprendizaje}

\subsection{Descripción de la experiencia}

La experiencia consiste en la implementación de una unidad didáctica de probabilidad con un grupo de alumnos, y se describe completamente en Beltrán-Pellicer (2016). La investigación llevada a cabo es exploratoria y descriptiva, de carácter naturalista (HERNÁNDEZ; FERNÁNDEZ; BAPTISTA, 2010), basada en la aplicación de los indicadores de la GVID obtenida sobre las anotaciones del diario del profesor.

La selección de la muestra fue intencional, atendiendo a la disposición del centro escolar y de la disponibilidad del investigador, primer autor de este artículo y que también actúa como profesor. Se trata de un grupo de dieciocho alumnos de $3^{\circ}$ de ESO (14-15 años), durante el curso académico 2015/2016, que cursan sus estudios en un Instituto de Educación Secundaria de carácter público, en España. En la normativa del centro educativo (programación didáctica) se indica la referencia del libro de texto (COLERA et al., 2007), que servirá como base para el desarrollo de la unidad y que se complementa con otras actividades.

\subsection{Ejemplo de reflexión sobre la valoración de la idoneidad de la secuencia}

Nos centramos, aquí, en aquellas facetas de la idoneidad que se han visto particularizadas en el proceso de elaboración de la GVID para probabilidad: la epistémica y la cognitiva. En el trabajo de Beltrán-Pellicer y Godino (2017) se muestra un análisis reflexivo del resto de las facetas. No obstante, dada la interrelación entre ellas, se destacan aquí sus interacciones más destacables.

\subsubsection{Idoneidad epistémica}

La observación muestra la realización de una trayectoria epistémica óptima, introduciendo, de forma articulada, los diferentes significados de la probabilidad (BATANERO, 2005) y empleando experiencias reales y simulaciones (GODINO et al., 1987). 
Sin embargo, no se ha encontrado anotaciones que evidencien que se hayan propuesto situaciones de generación de problemas sobre fenómenos aleatorios, sino que todas han venido dadas de antemano por el profesor.

En el plano lingüístico, la observación ha permitido comprobar que se han trabajado múltiples registros para describir las experiencias y situaciones en las que interviene el azar. Sin embargo, no se ha detectado la utilización de tablas de contingencia como contrapunto a los diagramas en árbol, y apenas se han recogido anotaciones que enfaticen la representación conjuntista. La planificación original a cinco sesiones, más la prueba escrita, no dejaba espacio para incluir el tratamiento de un número mayor de representaciones, así que se optó por los diagramas en árbol.

La recomendación de mejora, en este sentido, consiste en planificar el diseño para diez sesiones reales, introduciendo más registros y representaciones, así como proponer tareas donde los alumnos generen sus propias situaciones. El compromiso entre idoneidad epistémica-ecológica y mediacional-interaccional surge, entonces, de forma natural; puesto que, si enriquecemos esta unidad didáctica dándole más recursos temporales, habrá otras unidades que deberán tratarse en un menor número de sesiones.

Un compromiso similar tendría lugar si se decide aumentar el tiempo que deben dedicar los alumnos a las tareas propias de la unidad fuera del aula. Es decir, trasladar gran parte del trabajo cognitivo fuera del tiempo lectivo. Además de que ecológicamente se resentiría la idoneidad del proceso de instrucción; no parece que, con este tipo de alumnado, que escasamente ha asumido la responsabilidad del proceso de estudio, se consiguiera una asunción real de los objetivos de aprendizaje.

En lo que respecta a la adaptación socio-cultural del proceso de estudio implementado, ya se ha comentado en la faceta afectiva que es muy llamativo el hecho de que gran parte del alumnado asista a clases particulares de refuerzo o academias de repaso, a pesar de que cognitivamente no precisen de ello. En una entrevista informal, una madre señala, de forma sincera, que como lo hacen muchas familias de su entorno, ellos también han terminado por llevar a su hijo a profesor particular de cuando en cuando.

Las principales actividades que realizan en esos refuerzos son la realización de deberes y la preparación de exámenes. Ambas acciones inciden de forma negativa en el fomento de actitudes de responsabilidad y autonomía para el estudio, además de que los enfoques didácticos difieren. En algunos casos, se favorece la disrupción en clase, porque se limitan a lo que puedan hacer en esos refuerzos. 
La decisión de diseño de limitar el campo de situaciones combinatorias a contextos en donde el número de casos posibles es lo suficientemente pequeño como para poder ser desglosado mediante una tabla o un diagrama de árbol, ocasiona que no se hayan tratado apuestas, juegos de cartas como el póker o las máquinas de los casinos. Si se quiere mejorar este aspecto, el nuevo diseño debe incluir al menos una sesión para introducir técnicas de recuento algo más sofisticadas que las vistas, a modo de introducción del concepto de factorial de un número.

\subsubsection{Idoneidad cognitiva}

Se ha tenido en cuenta la posibilidad de que el grupo de alumnos apenas hubiese tratado contenidos del bloque de probabilidad y estadística. De hecho, en la primera sesión ya se evidenciaron sesgos en el razonamiento probabilístico (concepciones erróneas sobre las secuencias aleatorias) (KONOLD, 1989, 1991; LECOUTRE, 1992; SERRANO et al., 1998; TVERSKY; KAHNEMAN, 1974) y los propios alumnos comentaron que, prácticamente, no habían visto nunca probabilidad. Así, la secuencia de presentación de los contenidos ha seguido la trayectoria óptima, partiendo de una aproximación intuitiva (GODINO et al., 1987). Esto contribuye a una elevada idoneidad cognitiva, en cuanto a que el contenido está en la zona de desarrollo potencial de los alumnos.

En lo que respecta a las adaptaciones curriculares y la atención a las diferencias individuales, la idoneidad se ha visto resentida porque, a pesar de haber reservado bastante tiempo lectivo al trabajo indagativo por parejas, finalmente tuvo lugar una deriva importante hacia patrones de interacción totalmente dirigidos por el docente, en una enseñanza muy transmisiva. Las causas de esta desviación respecto al diseño hay que buscarlas en las actitudes y creencias del alumnado hacia el estudio y el trabajo en el aula (HANNULA et al., 2005), proponiendo como mejora el establecimiento de un nuevo contrato didáctico.

Es decir, si el docente pretende dar un peso importante a la interacción y al trabajo en clase, es algo que debería quedar claro ya desde la programación didáctica, estableciendo un mayor porcentaje para el trabajo en clase. Esto es algo que debe quedar especificado y justificado en la normativa del departamento, en la programación didáctica. Actualmente, la prueba escrita recibe el $90 \%$ de la calificación, hecho que suele considerarse normal (COLOMINA; ONRUBIA; NARANJO, 2000). Por lo tanto, es previsible un conflicto de tipo ecológico. 
Esto último enlaza con la baja idoneidad detectada en el apartado evaluativo de los aprendizajes logrados. Por una parte, la baja puntuación media de la prueba escrita indica que los objetivos de aprendizaje no han sido alcanzados por gran parte de los alumnos. Por otra, la importancia extrema que se le da a la prueba escrita en la programación es el comienzo de una compleja serie de interacciones entre facetas. El posible intercambio de información que tuviera lugar en las argumentaciones y discusiones de clase o en pequeños grupos sería un excelente instrumento de evaluación formativa. Ahora bien, al no verse apenas reflejado de forma directa en la calificación, el alumnado no se implica y el docente se ve obligado a cambiar el diseño.

La predominancia de la disrupción sobre otras acciones que debería realizar el alumnado incide de forma negativa sobre la faceta afectiva (BELTRÁN-PELLICER; GODINO, 2017). En el aspecto positivo, se han implementado situaciones en las que se subraya la utilidad del razonamiento probabilístico en la toma de decisiones.

\section{Consideraciones finales}

En esta investigación se ha destacado la importancia de la competencia de reflexión sobre la práctica docente. No en vano, la literatura considera que dicha competencia debe ser tenida en cuenta en los programas de formación del profesorado (LLINARES, 2012; PONTE et al., 2016; RAMOS-RODRÍGUEZ; FLORES; PONTE, 2017) y, de hecho, ciertos estudios señalan el bajo nivel de reflexión alcanzado por profesores en formación inicial (SECKEL; FONT, 2015).

En este sentido, ante la necesidad de contar con herramientas teórico-metodológicas que permitan involucrarse en los procesos formativos, el presente trabajo plantea la necesidad de elaboración de criterios e indicadores como base para la reflexión docente, aportando, así, a la línea de investigación de autores como Breda, Pino-Fan y Font (2017), Giacomone, Godino y Beltrán-Pellicer (2018). De esta forma, el propósito como investigadores fue presentar, con detalle, el proceso de elaboración de los indicadores de idoneidad didáctica para un tema específico, la probabilidad en niveles de educación secundaria.

Por otro lado, el propósito educativo fue poner en evidencia cómo estos indicadores permiten guiar la reflexión sobre una secuencia didáctica, identificando mejoras y compromisos de diseño que deberán ser tenidos en cuenta en futuras implementaciones.

Esta mirada a nivel macroscópico de la práctica docente debería ser una competencia 
del profesor de matemáticas (GODINO et al., 2017). En este sentido, los cursos y seminarios de formación de profesores deben incidir en la competencia para valorar el grado de adecuación de la práctica docente, tanto desde la autoevaluación como en sesiones de puesta en común con los compañeros de departamento. Esto abre una importante línea de investigación, dado que dichas acciones formativas se vienen experimentando ampliamente en los programas de maestría de diversas universidades españolas y latinoamericanas, como se puede ver en Breda, Font y Lima (2015).

\section{Agradecimientos}

Este trabajo se desarrolla en el marco del proyecto EDU2016-74848-P (FEDER, AEI), del Grupo FQM-126 (Junta de Andalucía) y dentro del Grupo «S119-Investigación en Educación Matemática» financiado por el Gobierno de Aragón y el Fondo Social Europeo.

\section{Referencias}

ALSINA, À.; DOMINGO, M. Idoneidad didáctica de un protocolo sociocultural de enseñanza y aprendizaje de las matemáticas. Revista Latinoamericana de Investigación en Matemática Educativa, México DF, v. 13, n. 1, p. 7-32, feb. 2010.

ARGUEDAS, C.; CONCARI, S.; GIACOMONE, B. La idoneidad didáctica de los laboratorios remotos como recursos para la enseñanza y aprendizaje de la física. Revista de Enseñanza de la Física, Córdoba, Argentina, v. 29, n. extra, p. 511-517, nov. 2017.

AROZA, C. J.; GODINO, J. D.; BELTRÁN-PELLICER, P. Iniciación a la innovación e investigación educativa mediante el análisis de la idoneidad didáctica de una experiencia de enseñanza sobre proporcionalidad. AIRES, Granada, España, v. 6, n. 6, p. 1-29, 2016.

BATANERO, C. Significados de la probabilidad en educación secundaria. Revista Latinoamericana de Investigación en Matemática Educativa, México DF, v. 8, n. 1, p. 247-263, 2005.

BATANERO, C. Understanding randomness: challenges for research and teaching. In: KRAINER, K.; VONDROVÁ, N. (Ed.). NINTH CONGRESS OF EUROPEAN SOCIETY FOR RESEARCH IN MATHEMATICS EDUCATION. 9., 2015. Prague. Proceedings of the Ninth Congress of European Society for Research in Mathematics Education. Prague: Charles University in Prague, Faculty of Education and ERME, 2015. p. 34-49.

BATANERO, C.; GODINO, J. D. Estocástica y su didáctica para maestros. Granada: Universidad de Granada, 2002.

BATANERO, C.; SÁNCHEZ, E. What is the nature of high school students' conceptions and misconceptions about probability? In: JONES, G. A. (Ed.). Exploring Probability in School. New York, NY: Springer, 2005. p. 241-266.

BELTRÁN-PELLICER, P. Evaluación de la Idoneidad Didáctica de una Experiencia de Enseñanza del Azar y Probabilidad en Tercer Curso ESO. Tesis (Maestría en Educación) - 
Facultad de Ciencias de la Educación, Universidad de Granada, Granada, 2016.

BELTRÁN-PELLICER, P.; GODINO, J. D. Aplicación de indicadores de idoneidad afectiva en un proceso de enseñanza de probabilidad en educación secundaria. Perspectiva Educacional, Viña del Mar, v. 56, n. 2, p. 92-116, 2017.

BLANCO-ÁLVAREZ, H.; FERNÁNDEZ-OLIVERAS, A.; OLIVERAS, M. L. Evaluación de una clase de matemáticas diseñada desde la etnomatemática. In: CONTRERAS, J. M.; ARTEAGA, P.; CAÑADAS, G. R.; GEA, M. M.; GIACOMONE, B.; LÓPEZ-MARTÍN, M. M. (Eds.). Actas del Segundo Congreso International Virtual sobre el Enfoque Ontosemiótico del Conocimiento y la Instrucción Matemáticos. Granada: CIVEOS, 2017, p. 1-9.

BLANCO, L.; GUERRERO, E.; CABALLERO, A. Cognition and affect in mathematics problem solving with prospective teachers. Mathematics Enthusiast, Missoula, MO, v. 10, n. 1/2, p. 335-364, 2013.

BRADBURY-HUAN, H. What is good action research? Why the resurgent interest? Action Research, Thousand Oaks, CA, v. 8, n. 1, p. 93-109, 2010.

BREDA, A.; FONT, V.; LIMA, V. M. R. A noção de idoneidade didática e seu uso na formação de professores de matemática. Jornal Internacional de Estudos em Educação Matemática, Londrina, v. 8 , n. 2 , p. $1-41,2015$.

BREDA, A.; FONT, V.; PINO-FAN, L. R. Criterios valorativos y normativos en didáctica de las matemáticas: el caso del constructo idoneidad didáctica. Bolema, Rio Claro, v. 32, n. 60, p. 255-278, abr. 2018.

BREDA, A.; PINO-FAN, L.; FONT, V. Meta Didactic-Mathematical Knowledge of Teachers: Criteria for the reflection and assessment on teaching practice. EURASIA Journal of Mathematics Science and Technology Education, London, v. 13, n. 6, p. 1893-1918, jun. 2017.

BROUSSEAU, G. Theory of didactical situations in mathematics. 1. ed. Dordrecht: Kluwer, 1997.

CARDANO, G. The book on games of chances. (Trabajo original publicado en 1663). 1. ed. New York: Holt, Rinehart y Winston, 1961.

CHAPUT, B.; GIRARD, J. C.; HENRY, M. Frequentist approach: Modelling and simulation in statistics and probability teaching. In: BATANERO, C.; BURRILL, G.; READING, C. (Ed.). Teaching Statistics in School Mathematics-Challenges for Teaching and Teacher Education. Netherlands: Springer, 2011. p. 85-95.

COBB, P.; BAUERSFELD, H. (Ed.). The emergence of mathematical meaning: Interaction in classroom cultures. 1. ed. Hillsdale, N.J.: Lawrence Erlbaum Associates Pub, 1995.

COLERA, J. et al. Matemáticas $3^{\circ}$ ESO. 1. ed. Madrid: Anaya, 2007.

COLOMINA, R.; ONRUBIA, J.; NARANJO, M. Las pruebas escritas y la evaluación del aprendizaje matemático en la educación obligatoria. Revista Electrónica Interuniversitaria de Formación del Profesorado, Murcia, v. 3, n. 2, p. 1-15, 2000.

CRUZ, A.; GEA, M.M.; GIACOMONE, B. Criterios de idoneidad epistémica para el estudio de la geometría espacial en educación primaria. In: CONTRERAS, J. M. et al. (Eds.), Actas del Segundo Congreso International Virtual sobre el Enfoque Ontosemiótico del Conocimiento y la Instrucción Matemáticos. Granada, España: CIVEOS, 2017, p. 1-10. 
CRUZ, A.; et al. Criterios de idoneidad cognitiva para el estudio de la geometría espacial en educación primaria. Actas del VIII Congreso Iberoamericano de Educación Matemática. Madrid, España: CIBEM, 2017, p. 1-8.

ELLIOT, J. La investigación-acción en educación. 1. ed. Madrid: Morata, 1990.

GAL, I. Towards "probability literacy" for all citizens: building blocks and instructional dilemmas. In: JONES, G. A. (Ed.). Exploring probability in school. Challenges for teaching and learning. New York: Springer, 2005. p. 39-64.

GELLERT, U.; BECERRA, R.; CHAPMAN, O. Research methods in mathematics teacher education. In: CLEMENTS, M. A. et al. (Ed.). THIRD INTERNATIONAL HANDBOOK OF MATHEMATICS EDUCATION, 3., 2013. New York. Third International Handbook of Mathematics Education. New York: Springer International, 2013. p. 327-360.

GIACOMONE, B.; GODINO, J. D.; BELTRÁN-PELLICER, P. Developing the prospective mathematics teachers' didactical suitability analysis competence. Educação e Pesquisa, São Paulo, v. 44, p. 1-21, may. 2018.

GODINO, J. D. Indicadores de idoneidad didáctica de procesos de enseñanza y aprendizaje de las matemáticas. Cuadernos de Investigación y Formación en Educación Matemática, San José, v. 8, n. 11, p. 111-132, 2013.

GODINO, J. D.; BATANERO, C.; CAÑIZARES, M. J. Azar y probabilidad. Madrid: Editorial Síntesis, 1987.

GODINO, J. D.; BATANERO, C.; FONT, V. Un enfoque ontosemiótico del conocimiento y la instrucción matemática. ZDM: The International Journal on Mathematics Education, Berlin, v. 39, n. 1-2, p. 127-135, 2007.

GODINO, J. D.; et al. Análisis y valoración de la idoneidad didáctica de procesos de estudio de las matemáticas. Paradigma, Maracay Edo Aragua, v. 27, n. 2, p. 221-252, 2006.

GODINO, J. D.; et al. Enfoque Ontosemiótico de los Conocimientos y Competencias del Profesor de Matemáticas. Bolema, Rio Claro, v. 31, n. 57, p. 90-113, 2017.

GREEN, D. R. Probability concepts in school pupils aged 11-16 years. 1982. 476 p. Tesis (Doctor of Philosophy) - Loughborough University of Technology, Loughborough, 1982.

HANNULA, M. S. et al. Affect and mathematical thinking. Role of beliefs, emotions and other affective factors. In: BOSCH, M. et. al. FOURTH CONGRESS OF THE EUROPEAN SOCIETY FOR RESEARCH IN MATHEMATICS EDUCATION. 4., 2005. San Feliu de Guíxols. Proceedings of the Fourth Congress of the European Society for Research in Mathematics Education, San Feliu de Guíxols: FUNDEMI IQS - Universitat Ramon Llull, 2005. p. 163-284.

HART, L.; ALSTON, A.; MURATA, A. (Ed.). Lesson study research and practice in mathematics education. 1. ed. Dordrecht: Springer, 2011.

HERNÁNDEZ, R.; FERNÁNDEZ, C.; BAPTISTA, M. P. Metodología de la investigación. 5. ed. México: McGraw-Hill, 2010.

KONOLD, C. Informal conceptions of probability. Cognition and Instruction, London, v. 6, n. 1, p. 59-98, 1989. 
KONOLD, C. Understanding students' beliefs about probability. In: GLASERSFELD, E. von (Ed.). Radical Constructivism in Mathematics Education. 1. ed. Dordrecht: Kluwer, 1991, p. 139-156

LECOUTRE, M. P. Cognitive models and problem spaces in «purely random» situations. Educational Studies in Mathematics, Dordrecht, v. 23, n. 1, p. 557-568, 1992.

LECOUTRE, M. P.; DURAND, J. L. Jugements probabilistes et modèles cognitifs: étude d'une situation aléatoire. Educational Studies in Mathematics, Dordrecht, v. 19, n. 3, p. 357-368, 1988.

LLINARES, S. Construcción de conocimiento y desarrollo de una mirada profesional para la práctica de enseñar matemáticas en entornos en línea. Avances de Investigación en Educación Matemática, Madrid, v. 1, n. 2, p. 53-70, 2012.

MCLEOD, D. Affective issues in mathematical problem solving: Some theoretical considerations. Journal for Research in Mathematics Education, Reston, VA, v. 19, n. 2, p. 134-141, 1988.

PARADA, S.; PLUVINAGE, F. Reflexiones de profesores de matemáticas sobre aspectos relacionados con su pensamiento didáctico. Revista Latinoamericana de Investigación en Matemática Educativa, México DF, v. 17, n. 1, p. 83-113, 2014.

PASCAL, B. Correspondance avec Fermat. En Oeuvres Complètes. (Trabajo original publicado en 1654). 1. ed. París: Seuil, 1963.

PIAGET, J.; INHELDER, B. La genése de l'idée de hasard chez l'enfant. 1. ed. Paris: Presses Universitaires de France, 1951.

POCHULU, M. D.; FONT, V.; RODRÍGUEZ, M. Desarrollo de la competencia en análisis didáctico de formadores de futuros profesores de matemática través del diseño de tareas. Revista Latinoamericana de Investigación en Matemática Educativa, México DF, v. 19, n. 1, p. 71-98, 2016.

PONTE, J. P. et al. Lesson Study as a Professional Development Process of Mathematics Teachers. Bolema, Rio Claro, v. 30, n. 56, p. 868-891, sep. 2016.

RAMOS-RODRÍGUEZ, E.; FLORES, P.; PONTE, J. P. An Approach to the Notion of Reflective Teacher and Its Exemplification on Mathematics Education. Systemic Practice and Action Research, New York, v. 30, n. 1, p. 85-102, feb. 2017.

ROBLES, M. G.; TELLECHEA, E.; FONT, V. Una propuesta de acercamiento alternativo al teorema fundamental del cálculo. Educación Matemática, México DF, v. 26, n. 2, p. 69-109, ago. 2014.

SCHÖN, D. The reflective practitioner: how professionals think in action. 1. ed. Nueva York: Basic Books, 1983.

SECKEL, M. J.; FONT, V. Competencia de reflexión en la formación inicial de profesores de matemática en Chile. Práxis Educacional, Vitória da Conquista, v. 11, n. 19, p. 55-75, 2015.

SERRANO, L. et al. Heurísticas y sesgos en el razonamiento probabilístico de los estudiantes de secundaria. Educación Matemática, México, v. 10, n. 1, p. 7-25, 1998.

TVERSKY, A.; KAHNEMAN, D. Judgment under uncertainty: Heuristics and biases. Science, Washington DC, v. 185, n. 4157, p. 1124-1131, 1974. 
WILLIAMS, R. J.; CONNOLLY, D. Does learning about the mathematics of gambling change gambling behavior? Psychology of Addictive Behaviors, Washington, DC, v. 20, n. 1, p. 62-68, 2006.

Submetido em 24 de Julho de 2017. Aprovado em 08 de Janeiro de 2018. 